OPEN ACCESS

Edited by:

David L. Moyes,

King's College London, United Kingdom

Reviewed by:

Todd B. Reynolds,

The University of Tennessee,

Knoxville, United States

Teresa Zelante,

University of Perugia, Italy

*Correspondence:

Selinda J. Orr

S.Orr@qub.ac.uk

Specialty section: This article was submitted to

Infectious Diseases,

a section of the journal

Frontiers in Microbiology

Received: 24 November 2020 Accepted: 08 February 2021 Published: 25 February 2021

Citation:

Thompson A, da Fonseca DM, Walker L, Griffiths JS, Taylor PR,

Gow NAR and Orr SJ (2021) Dependence on Mincle and Dectin-2 Varies With Multiple Candida Species

During Systemic Infection.

Front. Microbiol. 12:633229. doi: 10.3389/fmicb.2021.633229

\section{Dependence on Mincle and Dectin-2 Varies With Multiple Candida Species During Systemic Infection}

\author{
Aiysha Thompson 1,2, Diogo M. da Fonseca ${ }^{1,3}$, Louise Walker ${ }^{4}$, James S. Griffiths ${ }^{1,5}$, \\ Philip R. Taylor ${ }^{1,2}$, Neil A. R. Gow ${ }^{4,6}$ and Selinda J. Orr ${ }^{1,3 *}$ \\ 1 Division of Infection and Immunity, Systems Immunity Research Institute, Cardiff University School of Medicine, Cardiff, \\ United Kingdom, ${ }^{2}$ UK Dementia Research Institute, Cardiff, United Kingdom, ${ }^{3}$ School of Medicine, Dentistry and Biomedical \\ Science, Wellcome Wolfson Institute for Experimental Medicine, Queen's University Belfast, Belfast, United Kingdom, \\ ${ }^{4}$ Aberdeen Fungal Group, University of Aberdeen, Aberdeen, United Kingdom, ${ }^{5}$ Faculty of Dentistry, Oral \& Craniofacial \\ Sciences, Centre for Host-Microbiome Interactions, King's College London, London, United Kingdom, ${ }^{6}$ Medical Research \\ Council Centre for Medical Mycology, University of Exeter, Exeter, United Kingdom
}

More than 95\% of invasive Candida infections are caused by four Candida spp. (C. albicans, C. glabrata, C. tropicalis, C. parapsilosis). C-type lectin-like receptors (CLRs), such as Dectin-1, Dectin-2, and Mincle mediate immune responses to C. albicans. Dectin-1 promotes clearance of C. albicans, C. glabrata, C. tropicalis, and C. parapsilosis, however, dependence on Dectin-1 for specific immune responses varies with the different Candida spp. Dectin-2 is important for host immunity to C. albicans and C. glabrata, and Mincle is important for the immune response to C. albicans. However, whether Dectin-2 drives host immunity to C. tropicalis or C. parapsilosis, and whether Mincle mediates host immunity to C. glabrata, C. tropicalis or C. parapsilosis is unknown. Therefore, we compared the roles of Dectin-2 and Mincle in response to these four Candida spp. We demonstrate that these four Candida spp. cell walls have differential mannan contents. Mincle and Dectin-2 play a key role in regulating cytokine production in response to these four Candida spp. and Dectin-2 is also important for clearance of all four Candida spp. during systemic infection. However, Mincle was only important for clearance of $C$. tropicalis during systemic infection. Our data indicate that multiple Candida spp. have different mannan contents, and dependence on the mannan-detecting CLRs, Mincle, and Dectin-2 varies between different Candida spp. during systemic infection.

Keywords: Mincle, Dectin-2, Candida, CLR, fungal

\section{INTRODUCTION}

Candida spp. are the second most common agents of human fungal infection after dermatophytes (Brown et al., 2012). The vast majority of Candida infections (candidiasis) are superficial and pose no serious threat to immunocompetent individuals, however, recurrent mucosal infections impart a significant and widespread morbidity affecting more than 100 million women each year (Kullberg and Arendrup, 2015; Denning et al., 2018). Although invasive candidiasis is much less frequent 
than other superficial mycoses, it is associated with high mortality rates (Yapar, 2014; Mccarty and Pappas, 2016; Pappas et al., 2018). Indeed, invasive candidiasis is a worrying health concern as it is estimated to affect 250,000 people every year with associated mortality ranging from 46 to $75 \%$ (Brown et al., 2012; Bongomin et al., 2017), This high mortality stems mainly from the fact that current antifungal therapies for invasive candidiasis are limited, often administered late in the course of infection, and because the patients are often suffering from one or more predisposing factors (Zaoutis et al., 2005; Lockhart, 2014; Pappas et al., 2018; Sam et al., 2018). Immunotherapy as a potential treatment for invasive candidiasis has yet to be developed, however, this would require an in-depth understanding of the anti-fungal immune response (Segal et al., 2006; van de Veerdonk et al., 2010; Sam et al., 2018).

C. albicans is the most virulent and frequently isolated pathogen from patients with invasive candidiasis, accounting for $50-70 \%$ of all infections (Pfaller and Diekema, 2007; Guinea, 2014), however, invasive infections caused by other non-albicans Candida spp., such as C. parapsilosis, C. glabrata, and C. tropicalis and recently $C$. auris have been rising over the past decades (Arendrup et al., 2002; Yapar, 2014; Lamoth et al., 2018), and pose an emerging health concern. Nevertheless, most research tends to focus on C. albicans as a model pathogen of candidiasis, with much less research on other clinically relevant Candida spp.

Innate immune cells deploy several molecular mechanisms to control Candida proliferation and dissemination into host tissues, and to initiate adaptive immunity in order to ultimately curtail the infection. Innate phagocytes, such as macrophages and dendritic cells (DCs) recognize Candida spp. via an array of pattern-recognition receptors (PRRs), such as C-type lectin-like receptors (CLRs) and Toll-like receptors (TLRs) (Patin et al., 2019). These receptors sense pathogen-associated molecular patterns (PAMPs) mainly present in the fungal cell wall, the outermost structure of the Candida cell. The CLR, Dectin-1 was long identified as the main fungal $\beta$-(1,3)-glucanbinding PRR (Brown et al., 2002; Taylor et al., 2007), and was shown to be involved in the activation of protective immune mechanisms against $C$. albicans and C. glabrata via phagocytosis, the respiratory burst, neutrophil extracellular traps release, cytokine/chemokine secretion, recruitment of inflammatory cells and $\mathrm{T}$ cell response activation (Taylor et al., 2007; Hardison and Brown, 2012; Branzk et al., 2014; Chen et al., 2017). Dectin1 has been shown to contribute to host resistance against multiple forms of C. albicans-driven candidiasis (Ferwerda et al., 2009; Plantinga et al., 2009, 2010), however, we recently showed that the requirement for Dectin-1 for specific immune responses to Candida is species-dependent. We found that Dectin-1 abrogation significantly increases mortality in mice during C. albicans-induced systemic candidiasis but does not affect survival following infection with C. glabrata, C. tropicalis or C. parapsilosis (Thompson et al., 2019). However, fungal burden is increased in Dectin-1 KO mice following infection with all four Candida spp. (Thompson et al., 2019).

The outer cell wall of Candida is dominated by a layer of highly mannosylated cell wall proteins for which the mannan component determines the majority of the molecular mass. Mannans represent a shield that hides the inner glucan layer from recognition by Dectin-1, whilst innate cells recognize a range of mannans in the outer cell wall via a number of CLRs (Gow et al., 2011; Yadav et al., 2020). Mannans can be linked to cell wall proteins via amide linkages ( $N$-mannan) or ether linkages [O-mannan or via phosphodiester linkages to $N$ - or O-mannan (phosphomannan)] (Netea et al., 2006; Hall and Gow, 2013). Therefore, host cells rely on various mannan-binding receptors for their recognition including the mannose receptor, DC-SIGN, Galectin-3, Dectin-2, and Mincle. The ligands for these mannan-binding CLRs can either be superficial or buried within the inner cell wall (Vendele et al., 2020). Dectin-2 was shown to bind mannose-rich structures present in the Candida cell wall (McGreal et al., 2006; Sato et al., 2006), and to contribute to host protection during systemic infections with C. albicans and C. glabrata (Ifrim et al., 2014, 2016; Haider et al., 2019; Thompson et al., 2019). In C. albicans infections, Dectin-2 was shown to contribute toward the generation of protective Th1 and Th17 responses mainly by promoting production of IL-12p40, IL-1 $\beta$, and IL-23 by DCs (Robinson et al., 2009; Saijo et al., 2010). Mincle can bind $\alpha$-mannose residues present in fungal cell wall mannans (Taylor et al., 2005) and also acts as a death sensor by recognizing SAP130, an alarmin released by dying host cells that induces neutrophil infiltration into damaged tissues (Yamasaki et al., 2008). Although it was shown to have a redundant role in C. albicans phagocytosis by macrophages, Mincle is recruited to macrophage phagocytic synapse sites, and was shown to be involved in TNF production (Wells et al., 2008). Dependence on Mincle for resistance against C. albicans-driven systemic candidiasis appears to be strain-specific, as Mincle was reported to be necessary for controlling C. albicans 3630 clearance in the kidneys (Wells et al., 2008), but we recently showed that it plays a redundant role in conferring resistance toward C. albicans SC5314 (Thompson et al., 2019). Indeed, other authors have shown that Mincle does not bind multiple Candida spp., including C. albicans (Yamasaki et al., 2008), making it difficult to ascertain its importance for host protection during candidiasis.

Here we compare the cell wall composition of the four main Candida spp. responsible for the vast majority of invasive candidiasis (C. albicans, C. glabrata, C. tropicalis, and $C$. parapsilosis) and determine the role of Mincle and Dectin-2 in response to these different pathogens in a systemic model of infection.

\section{MATERIALS AND METHODS}

\section{Mice}

C57BL/6J (WT), Mincle KO (Clec4e $\left.e^{-/-}\right)$, and Dectin-2 KO $\left(\mathrm{Clec} 4 n^{-/-}\right)$mice were maintained and handled according to institutional and U.K. Home Office guidelines. Animals used were age- and gender-matched. In accordance with the 3Rs to reduce mouse numbers, wherever possible the same WT mice were used as controls for multiple $\mathrm{KO}$ mice strains in concurrent experiments. Where the same controls have been used for different figures, this is highlighted in the figure legend. 


\section{Ethics Statement}

All in vivo experimental procedures were approved by and performed in strict accordance to both Cardiff University's Animal Welfare and Ethical Review Body and the U.K. Home Office. Animal care and use adhered to the Animals (Scientific Procedures) Act 1986.

\section{Reagents}

IFN- $\gamma$ and IL-17 ELISAs were obtained from R\&D. IL-1 $\beta$, IL6, IL-10, IL-12p40, and TNF ELISAs were obtained from Life Technologies. M-CSF and GM-CSF used for bone marrow cell differentiation were obtained from Peprotech. Greiss reagent was purchased from Sigma.

\section{Preparation of Candida Cultures}

Candida albicans SC5314 reference strain was obtained from ATCC. The clinical isolates Candida glabrata SCS74761, Candida tropicalis SCS74663, Candida parapsilosis SCSB588, were kindly donated by Dr. Donna MacCallum (University of Aberdeen). Candida spp. were propagated on YPD agar plates overnight at $30^{\circ} \mathrm{C}$, then grown in YPD broth for $\sim 16 \mathrm{~h}$ at $30^{\circ} \mathrm{C}$ in a shaking incubator. Candida spp. were washed three times with PBS with centrifugation at $350 \times \mathrm{g}$, for $5 \mathrm{~min}$, and then resuspended at the appropriate concentration for experimental assays.

\section{Candida Preparation for TEM, Cell Wall Thickness, Mannan Fibril Length, and Alcian Blue Assay}

High-pressure freezing-freeze substitution transmission electron microscopy (TEM) of Candida spp. was performed as previously described (Walker et al., 2018). Briefly, cells were snap-frozen in liquid nitrogen at high pressure using a Leica Empact highpressure freezer (Leica, Milton Keynes, United Kingdom) and fixed using a Leica AFS freeze substitution system. Samples were then processed in a Lynx tissue processor and embedded in TAAB812 (TAAB Laboratories, Aldermaston, United Kingdom) epoxy resin. One-hundred-nanometer sections were cut with a Leica Ultracut E microtome and stained with uranyl acetate and lead citrate. Samples were imaged with a JEOL 1400 plus transmission microscope (JEOL UK Ltd., Hertfordshire, United Kingdom) and imaging with an AMT UltraVUE camera (Deben, Suffolk, United Kingdom). Five measurements for cell wall thickness and mannan fibril length were taken per cell and 15 cells were measured for each Candida spp. Alcian Blue binding assay was used to determine the phosphomannan content of each Candida spp., as previously described (Hobson et al., 2004). Briefly, exponentially grown cells from each Candida spp. were collected, washed twice with milliQ water and diluted 1 in 10. Cell pellets were resuspended in $1 \mathrm{ml}$ of $30 \mu \mathrm{g} / \mathrm{ml}$ Alcian Blue and incubated at room temperature for $10 \mathrm{~min}$. The cell pellet was then collected and the OD $620_{\mathrm{nm}}$ of the supernatant was measured to determine the content of Alcian Blue. The concentration of Alcian Blue bound to cells of each Candida spp. was then calculated.

\section{Cell Culture}

Femurs and tibiae of mice were flushed with PBS to harvest bone marrow. Bone marrow cells were cultured for 6 days in DMEM supplemented with $10 \%$ heat inactivated fetal bovine serum, $5 \%$ heat inactivated horse serum, $2 \mathrm{mM}$ L-glutamine, $100 \mathrm{U} / \mathrm{ml}$ penicillin/streptomycin, $10 \mathrm{mM} \mathrm{HEPES}$ and $10 \mathrm{ng} / \mathrm{ml} \mathrm{M-CSF}$ to generate bone marrow-derived macrophages (BMDMs) (Patin et al., 2016). Bone marrow cells were cultured for 8 days in RPMI 1640 medium containing 10\% heat inactivated fetal bovine serum, $2 \mathrm{mM}$ L-glutamine, $100 \mathrm{U} / \mathrm{ml}$ penicillin/streptomycin, $10 \mathrm{mM}$ HEPES, 1\% NEAA, $1 \mathrm{mM}$ sodium pyruvate, $50 \mu \mathrm{M}$ $\beta$-mercaptoethanol and $10 \mathrm{ng} / \mathrm{ml}$ GM-CSF to generate bone marrow-derived dendritic cells (BMDCs).

\section{Cell Stimulations and Cytokine Assays}

Differentiated BMDMs were harvested using $8 \mathrm{mg} / \mathrm{ml}$ lidocaine, washed and resuspended in RPMI 1640 supplemented with $10 \%$ heat inactivated fetal bovine serum and $100 \mathrm{U} / \mathrm{ml}$ penicillin/streptomycin. BMDMs/BMDCs were plated in 96-well plates at a density of $1 \times 10^{5}$ cells/well and incubated overnight at $37^{\circ} \mathrm{C}$. Media was removed the following day, and cells were stimulated with $1 \times 10^{5}$ Candida CFUs/well for $24 \mathrm{~h}$ in a final volume of $200 \mu \mathrm{l}$ fresh media. $2.5 \mu \mathrm{g} / \mathrm{ml}$ amphotericin $\mathrm{B}$ was added $2 \mathrm{~h}$ after stimulation. Cell culture supernatants were harvested and cytokine levels were measured by ELISA assays, according to the manufacturers' protocols. Nitric oxide production was detected using the Griess test (Sun et al., 2003). Briefly, following in vitro stimulation of host cells with Candida spp., cell culture supernatants were harvested and incubated with equal volumes of Griess reagent for $10 \mathrm{~min}$. Absorbance was read at $540_{n m}$ using a Multiskan Spectrum plate reader (Thermo Fisher Scientific) and nitrite levels in samples were calculated from a standard curve generated using a serially diluted sodium nitrite solution.

\section{In vivo Candida spp. Infections}

Candida spp. was suspended in PBS and intravenously injected in mice in a volume of $100 \mu \mathrm{l}$. The Candida spp. dose administered varied between different experiments (outlined in figure legends). Mice were weighed daily and examined twice a day by using a predefined scoring system, with $20 \%$ body weight loss set as an additional humane endpoint. Mice were culled by $\mathrm{CO}_{2}$ asphyxiation and death was confirmed by posterior cervical dislocation. At the end of each experiment blood was collected by cardiac puncture and kidneys, brains and spleens were harvested as previously described (Patin et al., 2016). Serum was extracted from blood by centrifugation at $10,000 \mathrm{rpm}$ for $10 \mathrm{~min}$ at $4^{\circ} \mathrm{C}$ in serum tubes and used in ELISA assays for cytokine detection. The left kidney and right brain were homogenized in $1 \mathrm{~mL}$ PBS, homogenates were serially diluted and spotted on petri dishes containing $50 \mu \mathrm{g} / \mathrm{ml}$ chloramphenicol in YPD and incubated for $24-48 \mathrm{~h}$ at $30^{\circ} \mathrm{C}$. Colonies were counted and fungal burden was calculated as CFUs/g of organ. The spleen was homogenized and erythrocytes were lysed using ACK lysis buffer. Splenocytes were washed by centrifugation and resuspended in IMDM supplemented with $10 \%$ heat inactivated fetal bovine 
serum, $2 \mathrm{mM}$ L-glutamine, $100 \mathrm{U} / \mathrm{ml}$ penicillin/streptomycin, 50 $\mu \mathrm{M} \beta$-mercaptoethanol. Cells were plated at $1 \times 10^{6}$ cells/well and left unstimulated or stimulated with $2 \times 10^{6}$ CFUs/well Candida spp. for $48 \mathrm{~h}$ at $37^{\circ} \mathrm{C}$. $2.5 \mu \mathrm{g} / \mathrm{ml}$ amphotericin $\mathrm{B}$ was added $2 \mathrm{~h}$ after stimulation. After $48 \mathrm{~h}$, supernatants were collected and used for IFN- $\gamma$ and IL-17 ELISA assays.

\section{Statistical Methods}

Data were analyzed using GraphPad Prism. For statistical analysis of two groups, Student's $t$ test was performed, and for three or more groups, One-way ANOVA with Bonferonni's post-test or Two-way ANOVA with Bonferonni's post-test was used. Datasets were transformed by $\mathrm{Y}=\operatorname{sqrt}(\mathrm{Y}+0.5)$ when data did not follow a Gaussian distribution and analyzed by either ANOVA or Student's $t$-test, or non-parametric tests if normality was not achieved after transformation. One sample $t$-test was used if all the samples were zero in one group. Statistical significance was considered to be achieved when $p$-values were less than 0.05: ${ }^{*} p<0.05,{ }^{* *} p<0.01,{ }^{* * *} p<0.001,{ }^{* * * *} p<0.0001$. All data are presented as means \pm SEM.

\section{RESULTS}

\section{Candida spp. Display Differences in Their Cell Wall}

We compared the cell wall structure of C. albicans, C. glabrata, C. tropicalis, and C. parapsilosis by TEM of high-pressure frozen Candida. We observed significant differences in cell wall thickness of the inner wall in the following descending order: C. albicans, C. parapsilosis, C. glabrata, C. tropicalis (Figures 1A,B). Mannosylated protein fibrils extend out from the inner layer in the TEM images. The Candida spp. displayed decreasing mannan fibril length in the following order: C. albicans, C. parapsilosis $=$ C. tropicalis, C. glabrata (Figures 1A,C). We then analyzed the cell wall phosphomannan content using Alcian Blue binding. The Candida spp. displayed decreasing phosphomannan content in the following order: C. albicans, C. tropicalis $=$ C. parapsilosis, C. glabrata (Figure 1D). The significant differences in the Candida cell walls particularly in length/type of mannan structures present may affect the immune response mediated by CLRs, such as Dectin-2 and Mincle in response to these different clinically relevant Candida spp.

\section{Mincle and Dectin-2 Regulate Cytokine Responses to Multiple Candida spp. in BMDCs}

Mincle has been shown to mediate TNF production from BMDMs in response to C. albicans (Wells et al., 2008) while Dectin-2 has been shown to drive cytokine production from BMDCs in response to C. albicans mannans (Saijo et al., 2010) and from macrophages during systemic infection with C. glabrata (Ifrim et al., 2014, 2016). Here, we investigated the role of Mincle and Dectin-2 during cytokine production from BMDMs and BMDCs in response to four Candida spp. To investigate this,
BMDMs and BMDCs from WT and Mincle KO or WT and Dectin-2 KO mice were stimulated with C. albicans SC5314, C. glabrata SCS74761, C. tropicalis SCS74663, or C. parapsilosis SCSB5882. We did not observe any major roles for Mincle (Supplementary Figures 1A,C) or Dectin-2 (Supplementary Figures 1B,C) in regulating cytokine production or nitric oxide production in response to these four Candida spp. This is largely unsurprising as BMDMs do not basally express either Mincle or Dectin-2 (Supplementary Figure 2). However, both Mincle and Dectin-2 regulate cytokine production in response to multiple Candida spp. in BMDCs. Mincle mediates IL-12p40 production and inhibits IL-1 $\beta$ production in response to multiple Candida spp. (Figure 2A). While Dectin-2 promotes IL-12p40 and IL-10 production in response to multiple Candida spp. (Figure 2B). Neither CLR is required for nitric oxide production from BMDCs (Figure 2C). Therefore, Mincle and Dectin-2 regulate cytokine production from BMDCs in response to multiple Candida spp. but they are largely uninvolved in cytokine production from BMDMs.

\section{In vivo Clearance of $C$. tropicalis Is Partially Dependent on Mincle}

As Mincle regulates cytokine production in response to multiple Candida spp. we next sought to determine whether Mincle plays any roles during systemic infection with these different Candida spp. To this end, WT and Mincle KO mice were systemically infected with C. albicans, C. glabrata, C. tropicalis, or C. parapsilosis.

The infection doses used were previously determined (Thompson et al., 2019). One-week post-infection, Mincle KO mice showed reduced presence of C. glabrata within the brain and decreased clearance of $C$. tropicalis from the brain compared to WT mice (Figure 3A). The WT and Mincle KO mice did not display any other major differences in the early clearance of these four Candida spp. from the main target organs. Mincle KO mice also displayed a subtle elevation in serum IL-6 levels 1week post infection with $C$. tropicalis likely due to the increased fungal burden in these mice (Figure 3B). We did not observe any other major differences in serum cytokine levels between WT and Mincle KO mice following infection with these Candida spp. In addition, we tested the ability of WT and Mincle KO splenocytes to produce IFN- $\gamma$ and IL-17 upon antigen restimulation with live Candida spp. in the presence of amphotericin B. We did not observe any major differences in the ability of these splenocytes to produce IFN- $\gamma$ or IL-17. Finally, we examined the requirement for Mincle for controlling systemic infection with these Candida spp. over time. Mincle was largely unimportant for controlling and/or clearing systemic infection with these different Candida spp. However, similar to the early clearance of $C$. tropicalis, Mincle once again played a role in the clearance of C. tropicalis over time as the kidneys in Mincle KO mice displayed increased fungal burden compared to WT mice after 21 days (Figures 4A,B). Mincle is therefore relatively unimportant for controlling systemic Candida infections with the specific strains used in this study, however, it plays a role during systemic infection with C. tropicalis. 
A

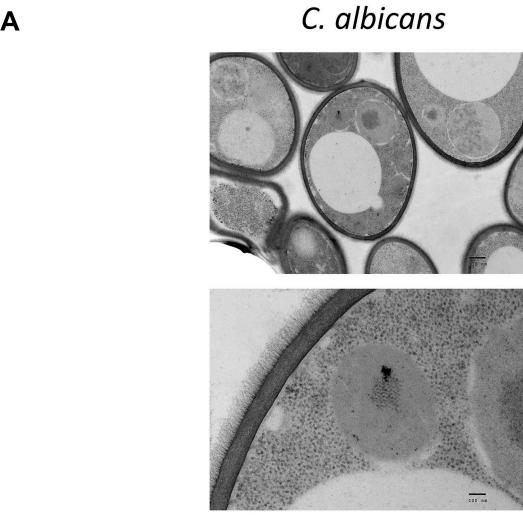

B

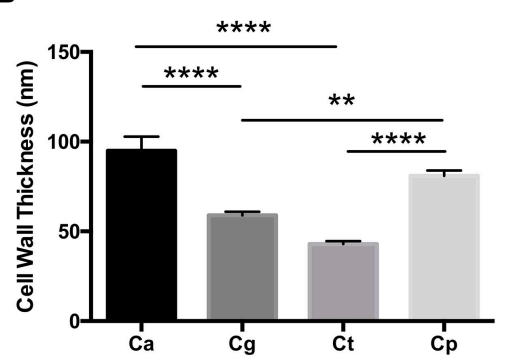

C. glabrata

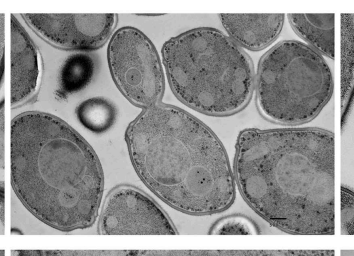

C. tropicalis
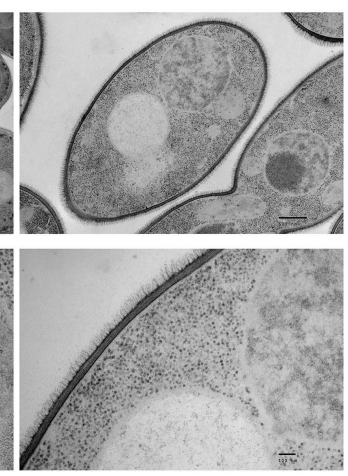

C. parapsilosis
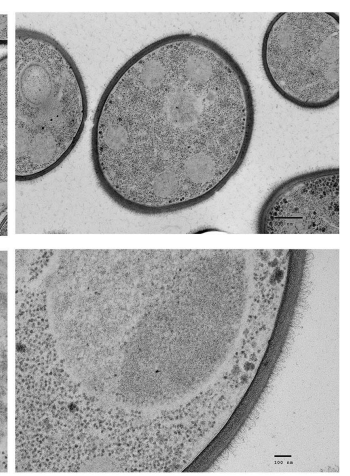

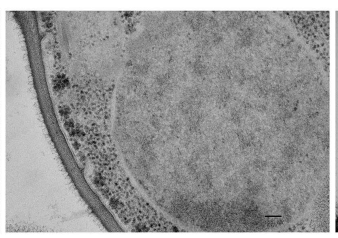

C

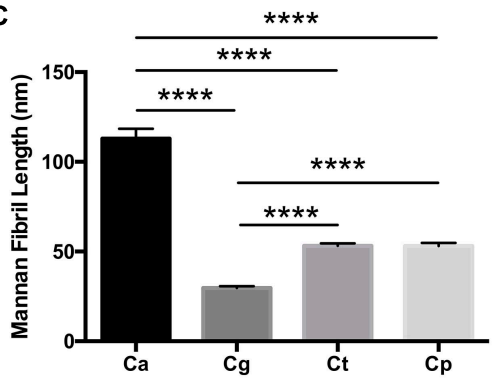

D

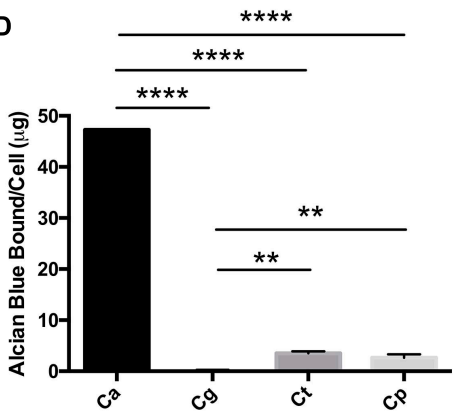

FIGURE 1 | Candida spp. yeast display differences in their cell wall. (A) Electron micrograph images showing the ultrastructure of Candida cells taken at 4,000× (upper panel) and 30,000 × (lower panel). Scale bars are $500 \mathrm{~nm}$ (upper panel) and $100 \mathrm{~nm}$ (lower panel). Images are representative of three separate preparations. (B,C) Cell wall thickness (B) and mannan fibril length (C) were measured from the TEM images. Graphs show the mean \pm SEM from 15 Candida cells (1-way ANOVA, Bonferroni's post-test). (D) Candida spp. were incubated in $30 \mu \mathrm{g} / \mathrm{ml}$ Alcian Blue for 10 min and the level of dye associated with the cell wall was measured by absorbance. Graph displays the mean amount of dye bound per cell \pm SEM from three independent experiments (One-way ANOVA with Bonferroni's post-test). ${ }^{* *} p<0.01,{ }^{* * *} p<0.0001$.

\section{Dectin-2 Is Important for in vivo Clearance of Multiple Candida spp.}

As Dectin-2 mediates cytokine production in response to multiple Candida spp. and it has been shown to play a role during infections with C. albicans (Saijo et al., 2010) and C. glabrata (Ifrim et al., 2014, 2016), we next analyzed the role for Dectin2 at different timepoints during systemic infection with these four clinically relevant Candida spp. To this end, WT and Dectin-2 KO mice were systemically infected with C. albicans, C. glabrata, C. tropicalis, or C. parapsilosis. One-week postinfection, Dectin-2 KO mice showed decreased clearance of C. parapsilosis from the brain compared to WT mice (Figure 5A). The WT and Dectin-2 KO mice did not display any other major differences in the early clearance of these four Candida spp. from the main target organs. We did not observe any major differences in serum cytokine levels between WT and Dectin-2 KO mice following infection with these Candida spp., however, Dectin-2 KO mice displayed a subtle elevation in serum IL-6 levels 1-week post infection with $C$. albicans or C. tropicalis (Figure 5B). Dectin-2 KO splenocytes produced more IFN- $\gamma$ upon antigen restimulation with live $C$. albicans and C. glabrata and increased IL-17 production following restimulation with C. albicans (Figure 5C). Finally, we examined the requirement for Dectin-2 for controlling systemic infection with these Candida spp. over time. Dectin-2 was important for clearance of all four Candida spp. (Figures 6A,B). Thus, Dectin2 is important during early infection with $C$. parapsilosis and at later timepoints for controlling systemic Candida infections with all four Candida spp.

\section{DISCUSSION}

In this study, we demonstrated that the cell wall thickness and mannan composition varied between $C$. albicans and three other clinically relevant, non-albicans species. We found that Mincle and Dectin-2 are involved in regulating cytokine production in response to all four Candida spp. However, in an in vivo model of systemic infection, we showed that Mincle is involved in clearance of C. tropicalis while Dectin-2 is involved in the clearance of all four Candida spp. Overall, we found that both Mincle and Dectin-2 play roles in response to different Candida spp. however this is host cell-, species- and likely strain- and site of infection-dependent.

As Mincle and Dectin-2 bind mannose/mannans in fungal cell walls, we hypothesized that differences in the cell wall mannan content between these four clinically relevant Candida spp. would determine whether Mincle or Dectin-2 are involved in the immune response to the different Candida spp. Mincle 

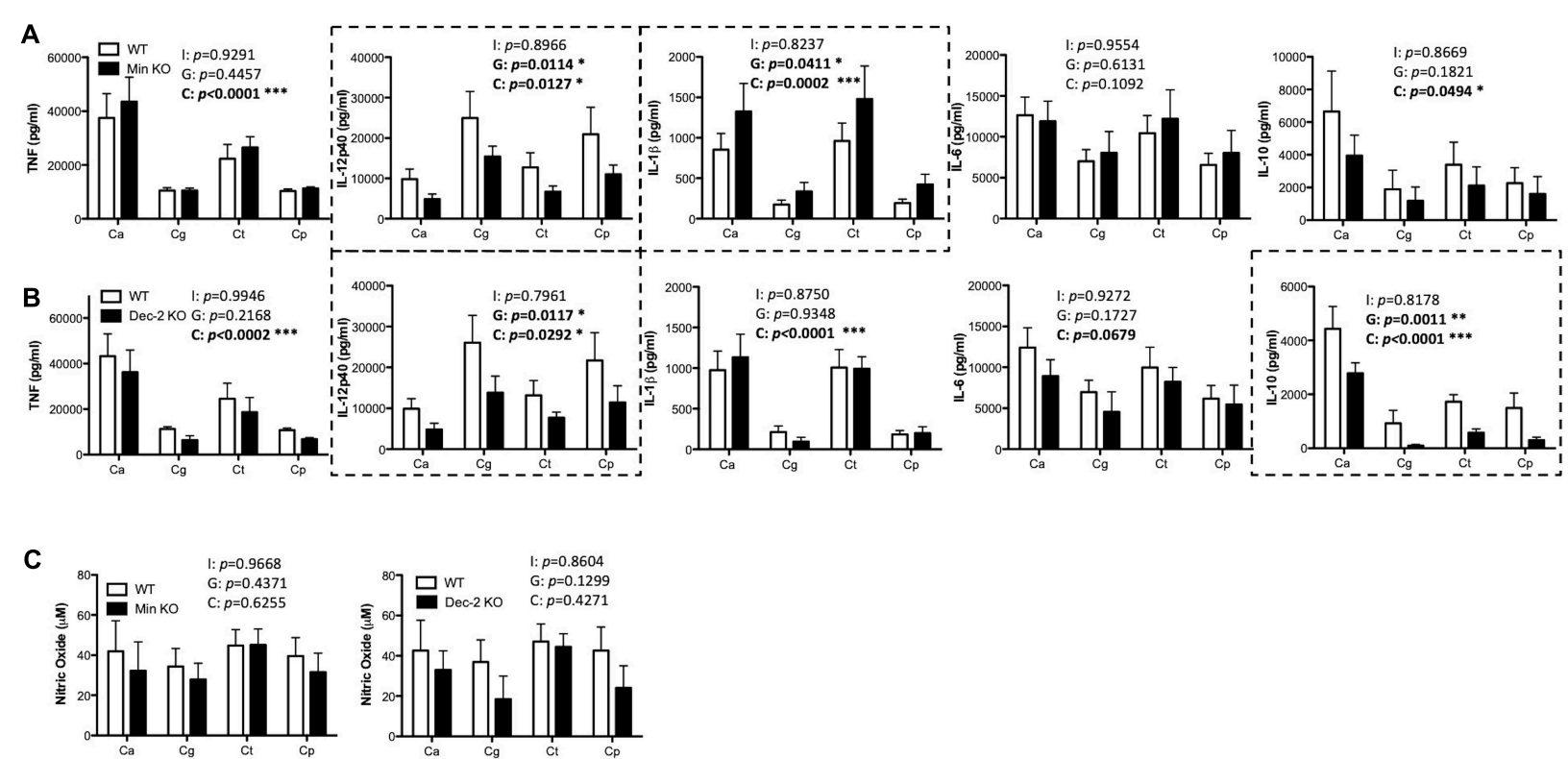

FIGURE 2 | Mincle and Dectin-2 regulate cytokine responses to multiple Candida spp. in BMDCs. (A-C) BMDCs from WT and Mincle KO mice (A,C) or from WT and Dectin-2 KO mice (B,C) were stimulated with C. albicans SC5314 (Ca), C. glabrata SCS74761 (Cg), C. tropicalis SCS74663 (Ct), and C. parapsilosis SCSB5882 (Cp) at a multiplicity of infection (MOI) of 1:1 (Candida sp.: BMDCs). Cytokine levels (A,B) and nitric oxide levels (C) in the supernatants were measured after $24 \mathrm{~h}$ incubation. Results are presented as means \pm SEM of 4 (A), 3-4 (B), or 3 (C) independent experiments (Two-way ANOVA with Bonferroni's post-test). I = Interaction between "Genotype" and "Candida" variables, G (Genotype) = impact of BMDC genotype (WT vs. Mincle KO; WT vs. Dectin-2 KO), C

(Candida) $=$ impact of different Candida spp. tested (albicans, glabrata, tropicalis, parapsilosis). Graphs where Genotype is significant have been surrounded by a dashed box. (B) WT data is the same as in (A) for three out of three replicates for TNF, three out of four replicates for IL-12p40, IL-1 $\beta$, and IL-6, and two out of three replicates for IL-10. (C) WT data in the right graph is the same as in the left graph for two out of three replicates. ${ }^{\star} p<0.05,{ }^{\star \star} p<0.01,{ }^{\star \star \star} p<0.001$.

has been shown to bind mannose (Lee et al., 2011) and Dectin-2 has been shown to bind high mannose structures (e.g., $\mathrm{Man}_{9} \mathrm{GlCNAc}_{2}$ ) (McGreal et al., 2006). Therefore, we compared the cell wall mannan content of these four clinically relevant Candida spp. Interestingly, we found large differences in the mannan fibril length and phosphomannan content. This would indicate significant differences in the $\mathrm{N}$-glycan structures on the surface of these four Candida spp. In agreement with our data, Nguyen et al. (2018) found that the molecular weights of Candida mannans decreased in the following order: C. albicans, C. tropicalis, C. glabrata. The predicted structures of these mannans differed substantially with much larger and more complex C. albicans mannans compared to the other Candida spp. (Nguyen et al., 2018). In addition, Shibata et al. (2007) showed that the mannan structure changed as $C$. albicans yeast transformed to hyphae. Moreover, several factors like different morphogenetic and morphological stages of Candida spp., the physicochemical properties of the substrate, such as the carbon source, $\mathrm{pH}$, oxygen levels, metal ion micronutrients and temperature, and also certain environmental stresses, such as the presence of different antifungal drugs were shown to alter carbohydrate synthesis and orientation in the fungal cell wall (Ballou et al., 2016; Pradhan et al., 2019; Vendele et al., 2020). Therefore, various factors will likely determine whether specific PRRs, such as Mincle and Dectin-2 are important for immune responses to these different Candida spp. These include: differences in the mannan content of the four Candida spp. when grown under the same conditions; the complexity of the mannans on the fungal cell wall at yeast, and likely hyphal stages; and the fact that some of these species do not form hyphae.

In this study, we next assessed whether Mincle or Dectin2 influenced cytokine release and nitric oxide production in response to these four Candida spp. In BMDCs, we found that Mincle mediates IL-12p40 production and regulates IL-1 $\beta$ production, while Dectin-2 drives production of IL-12p40 and IL-10 upon Candida spp. stimulation. Wells et al. (2008) reported that Mincle was involved in TNF production from macrophages following stimulation with C. albicans 3630 . We did not observe any defect in TNF production in Mincle KO BMDMs or BMDCs. The difference between our results and those previously published by Wells et al. (2008) could be due to the timepoints (1-2 h vs. $24 \mathrm{~h}$ ) or the strains of C. albicans (3630 vs. SC5314) used in the experiments. Dectin-2 was previously shown to be important for production of IL-12p40, TNF, IL-1 $\beta$, and IL-10 from BMDCs in response to C. albicans mannans (Saijo et al., 2010). In addition, naïve Dectin-2 KO peritoneal macrophages generally produced lower levels of TNF, IL-6, KC, IL-1 $\alpha$, and IL- $1 \beta$ compared to WT controls in response to $C$. albicans (Ifrim et al., 2016). However, cytokine production from naïve Dectin-2 $\mathrm{KO}$ peritoneal macrophages was similar to WT cells in response to C. glabrata (Ifrim et al., 2014). The different results could be due to the use of different cell types and whole $C$. albicans vs. C. albicans mannans. The altered IL-12p40 and IL- $1 \beta$ levels that we observed in Mincle KO BMDCs and the attenuated IL-12p40 


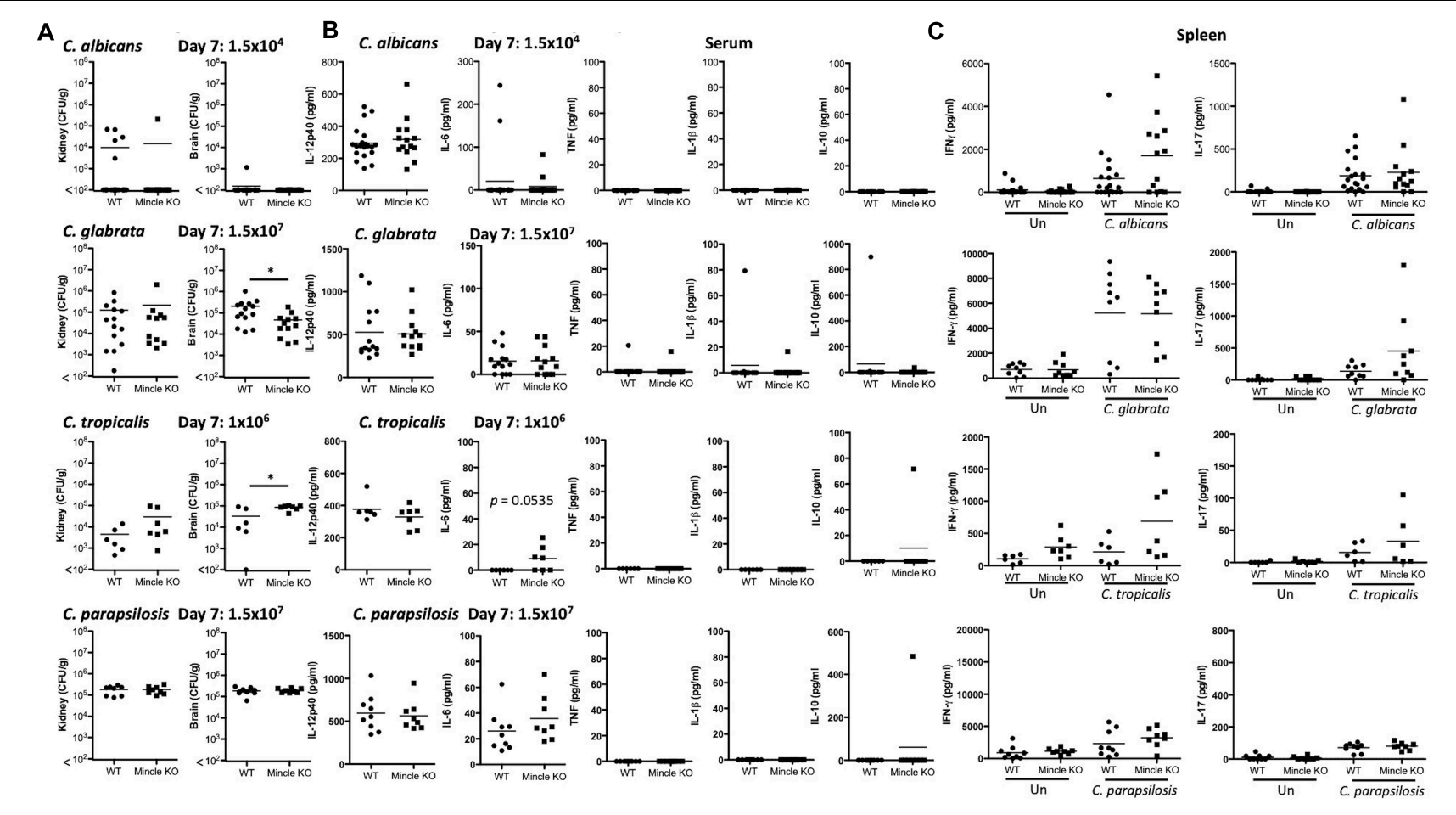

FIGURE 3 | Early clearance of $C$. tropicalis is partially dependent on Mincle. (A-C) WT and Mincle KO mice were intravenously infected with the indicated doses of C. albicans, C. glabrata, C. tropicalis, or C. parapsilosis for 7 days. (A) CFU in the kidneys and brains of mice at 7 days post-infection were determined. Mann-Whitney test (C. albicans kidney on transformed data; C. glabrata kidney and brain), One sample $t$-test (C. albicans brain), Student's $t$-test (C. tropicalis kidney; C. tropicalis brain on transformed data; C. parapsilosis kidney and brain). (B) Cytokine levels in the serum of mice at 7 days post-infection. Student's $t$-test (C. albicans IL-12p40 on transformed data; C. glabrata IL-12p40 and IL-6 on transformed data; C. tropicalis IL-12p40; C. parapsilosis IL-12p40 and IL-6 on transformed data), Mann-Whitney test (C. albicans IL-6 on transformed data; C. glabrata TNF, IL-1 $\beta$, and IL-10 on transformed data), One sample $t$-test (C. tropicalis IL-6 and IL-10; C. parapsilosis IL-10). (C) Splenocytes from infected mice were unstimulated or restimulated with live Candida spp. for 48 h and IFN- $\gamma$ and IL-17 levels were measured by ELISA. Kruskal Wallis with Dunn's post-test (C. albicans IFN- $\gamma$ and IL-17 on transformed data; C. glabrata IL-17 on transformed data; C. parapsilosis IFN- $\gamma$; C. parapsilosis IL-17 on transformed data), One-way ANOVA with Bonferroni's post-test (C. glabrata IFN- $\gamma$; C. tropicalis IFN- $\gamma$; C. tropicalis IL-17 on transformed data). (A-C) Graphs are the cumulative result of 2-4 independent experiments. Each symbol represents an individual mouse. Fifteen out of twenty WT mice in the C. albicans graphs, and five out of nine WT mice in the C. parapsilosis graphs are the same as Figure $\mathbf{4}$ in a previous publication from our group (Thompson et al., 2019). * $p<0.05$.

and IL-10 levels in Dectin-2 KO BMDCs would suggest that despite significant differences in the mannan levels/structures on the different Candida spp. that ligands for both these CLRs are present on C. albicans, C. glabrata, C. tropicalis, and C. parapsilosis. While we observed roles for Mincle and Dectin-2 in mediating/regulating cytokine production from BMDCs, this may differ for myeloid cell populations in target organs, such as the kidney or spleen.

As Mincle regulated cytokine production in vitro in response to these four Candida spp., we then assessed whether Mincle had any impact during systemic infection. We did not observe any major effect of Mincle deficiency on the clearance of C. albicans or C. parapsilosis either at early or late timepoints. This is in agreement with our previous findings (Thompson et al., 2019), while Wells et al. (2008) observed increased fungal burden in Mincle KO mice 5 days after systemic infection with C. albicans. The C. albicans strain, time point and dose differed between our studies and that by Wells et al. (2008). In our study, Mincle KO mice displayed slightly enhanced early clearance of C. glabrata from the brain, however, fungal burden was similar to WT at later timepoints and cytokine production was unaltered suggesting a minimal role. Interestingly, we found that although Mincle does not significantly influence survival, it plays a role in the clearance of $C$. tropicalis from the brain and kidneys of systemically infected mice at early and late timepoints. In previous studies with $C$. albicans, we have observed elevated IL-6 levels in the serum of mice with higher fungal burdens, and our data with Mincle KO mice suggest a similar trend during infection with C. tropicalis (Orr et al., 2013; Thompson et al., 2019). While we identified a role for Mincle in regulating cytokine production from all four Candida spp. in vitro, we only observed a major role for Mincle in regulating the clearance of $C$. tropicalis in vivo. Based on our findings we cannot rule out other roles for Mincle in vivo in response to these four Candida spp., however, our data indicate that while a ligand for Mincle may be present on all four Candida spp. tested, Mincle is only important for the clearance of C. tropicalis during systemic infection.

Similar to our experiments with Mincle, we then assessed whether Dectin-2 had any impact during systemic infection with all four Candida spp. We found that Dectin-2 is 
A
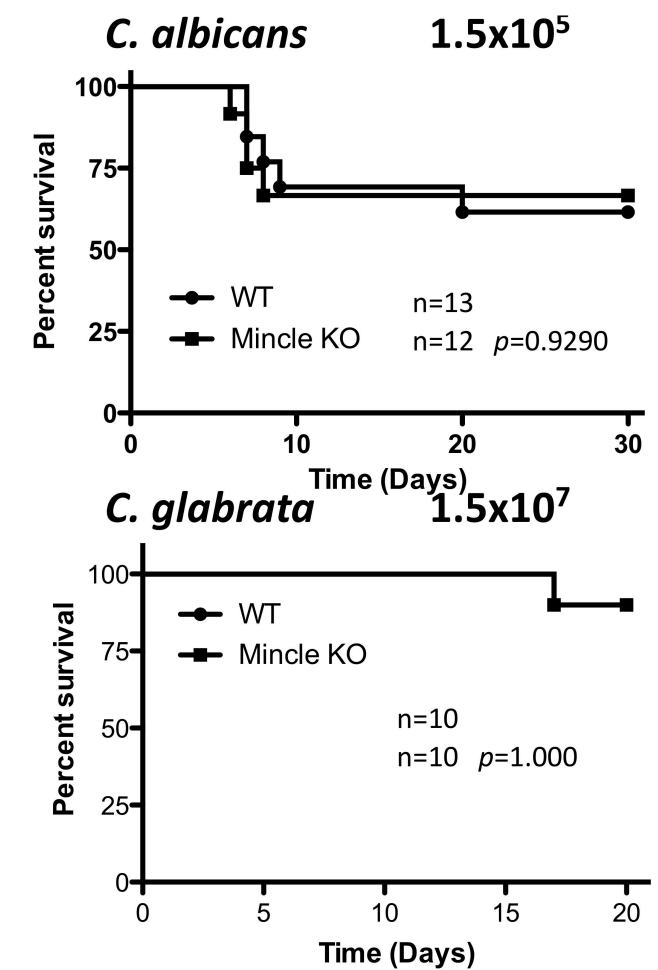

C. tropicalis

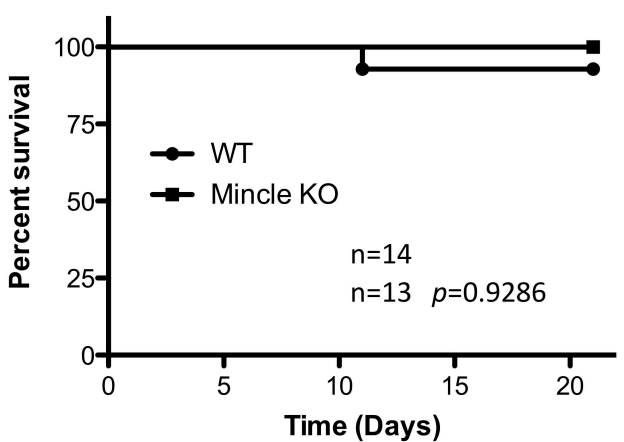

C. parapsilosis $1.5 \times 10^{7}$

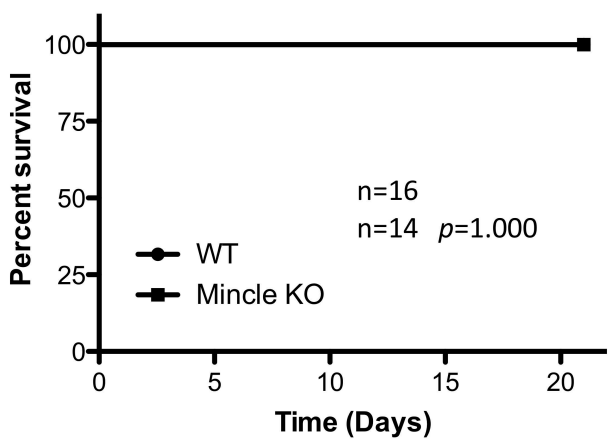

B

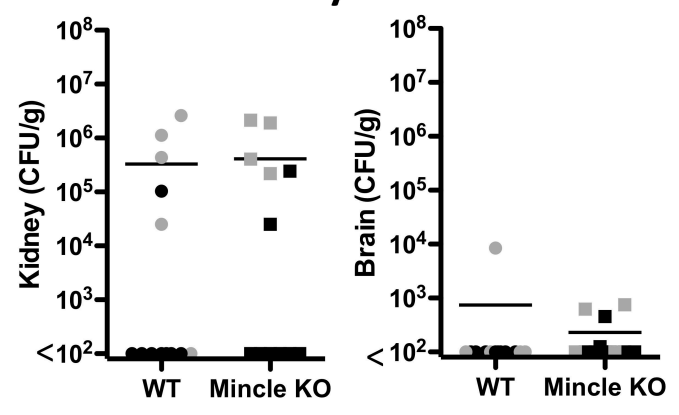

Day 20: $1.5 \times 10^{7}$
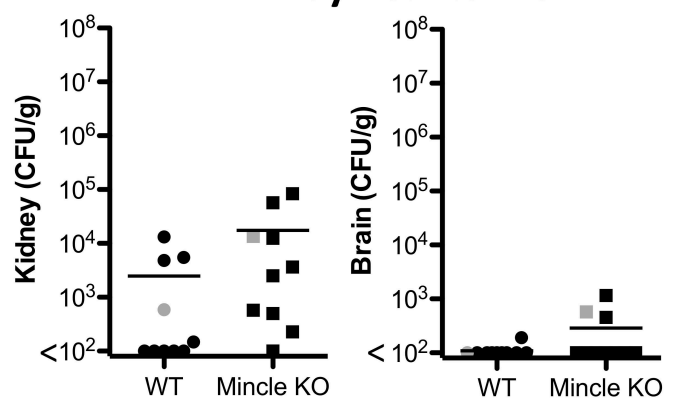

Day 21: $1 \times 10^{6}$
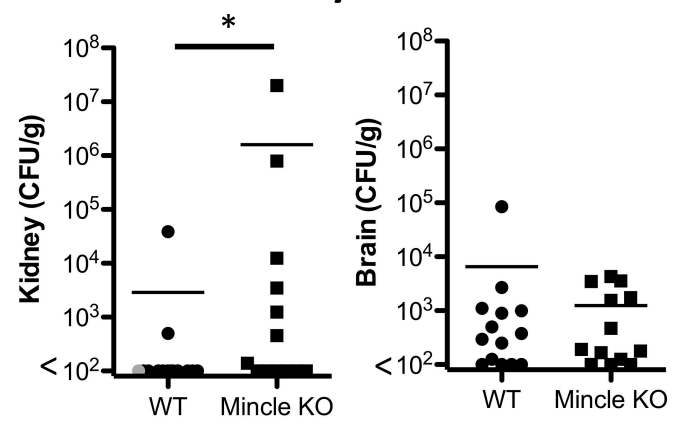

Day 21: $1.5 \times 10^{7}$

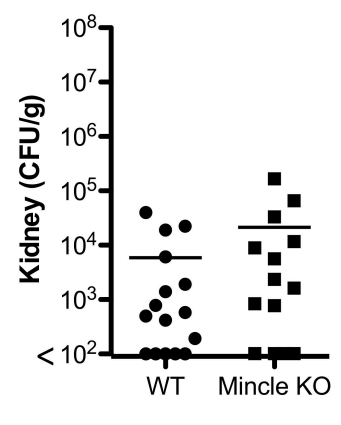

FIGURE 4 | Clearance of C. tropicalis is partially dependent on Mincle. (A) WT and Mincle KO mice were intravenously infected with the indicated doses of C. albicans for 30 days, C. glabrata for 20 days, C. tropicalis for 21 days, or C. parapsilosis for 21 days. Survival curves based on humane end-point of infected WT (filled circles) and Mincle KO mice (filled squares). Graphs are the cumulative result of 2-3 independent experiments. Log-rank test. (B) CFU in the kidneys of WT and Mincle KO mice at 20-30 days after infection (black symbols) or at time of death by humane end point (gray symbols). Graphs are the cumulative result of 2-3 independent experiments. Each symbol represents an individual mouse. Mann-Whitney test on transformed data (C. albicans kidney and brain; C. glabrata brain; C. tropicalis kidney and brain; C. parapsilosis kidney and brain), Student's t-test on transformed data (C. glabrata kidney). Four out of fourteen WT mice in the C. tropicalis graphs are the same as Figure $\mathbf{3}$ of a previous publication from our group (Thompson et al., 2019 ). ${ }^{\star} p<0.05$. 


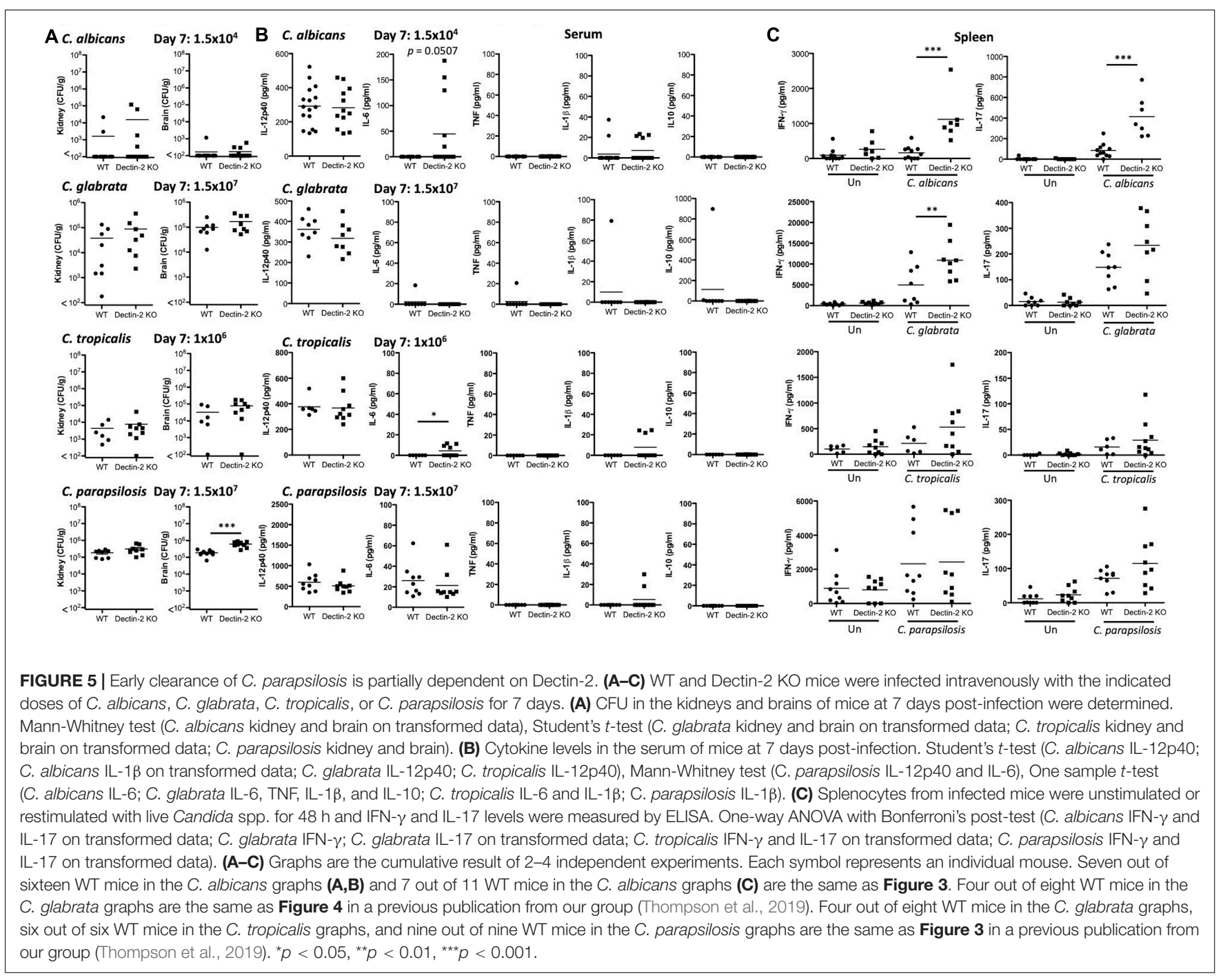

important for clearance of all Candida spp. tested, and that it significantly contributes to survival during C. albicans infection. The C. albicans and C. glabrata data is in agreement with previous findings (Saijo et al., 2010; Ifrim et al., 2014, 2016; Thompson et al., 2019). Serum IL-6 was also elevated to a significant or near significant level in Dectin-2 KO mice following infection with $C$. albicans and C. tropicalis similar to our previous observations (Orr et al., 2013; Thompson et al., 2019). Splenocytes from C. albicans- or C. glabrata-infected Dectin-2 KO mice displayed enhanced IL-17 and/or IFN- $\gamma$ production following restimulation with the same Candida sp. Ifrim et al. (2016) also showed similar enhanced T-cell associated cytokine production from Dectin-2 KO splenocytes following infection with $C$. albicans and restimulation with heat-killed C. albicans, although the time points differed from our study. However, in contrast to our findings, Ifrim et al. (2014) showed reduced T-cell associated cytokine production following infection with C. glabrata and restimulation with heat-killed C. glabrata. In addition, IL-17 production by splenocytes following infection with $C$. albicans was decreased with the addition of anti-Dectin-2
(Robinson et al., 2009). The experimental setup for each of these findings differ considerably, which could potentially explain some of the discrepancies. However, we believe that the enhanced IL-17 and/or IFN- $\gamma$ production from the Dectin- $2 \mathrm{KO}$ splenocytes in our study is likely associated with enhanced $\mathrm{T}$ cell exposure to the Candida spp. in vivo due to the inability of these mice to clear/control these infections. Saijo et al. (2010) showed reduced $\mathrm{T}$-cell associated cytokine production from purified naïve Dectin$2 \mathrm{KO} \mathrm{CD}^{+} \mathrm{T}$ cells following stimulation with supernatants from BMDCs cultured with C. albicans indicating an inability of Dectin-2 KO T cells to appropriately respond to C. albicans. Therefore, our data indicate that Dectin-2 is important for the clearance of all four Candida spp., similar to our in vitro cytokine findings, however, roles for Dectin-2 in specific responses in vivo during systemic infection varies with Candida spp. Our data does not rule out additional roles for Dectin-2 in mediating production of other cytokines in response to these four Candida species or at different sites of infection.

At the start of this study, we postulated that differences in host immune responses and consequent in vivo fungal 
A

\section{C. albicans}

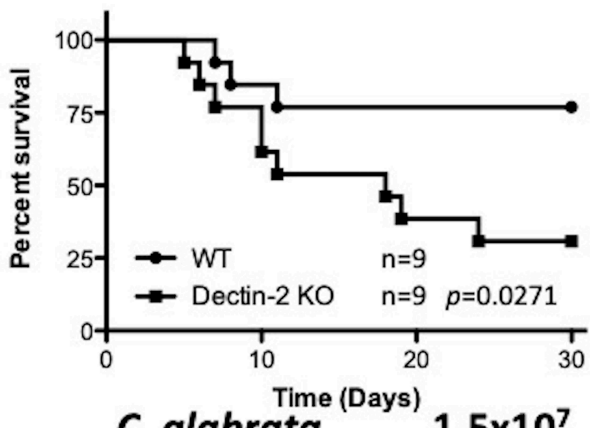

C. glabrata
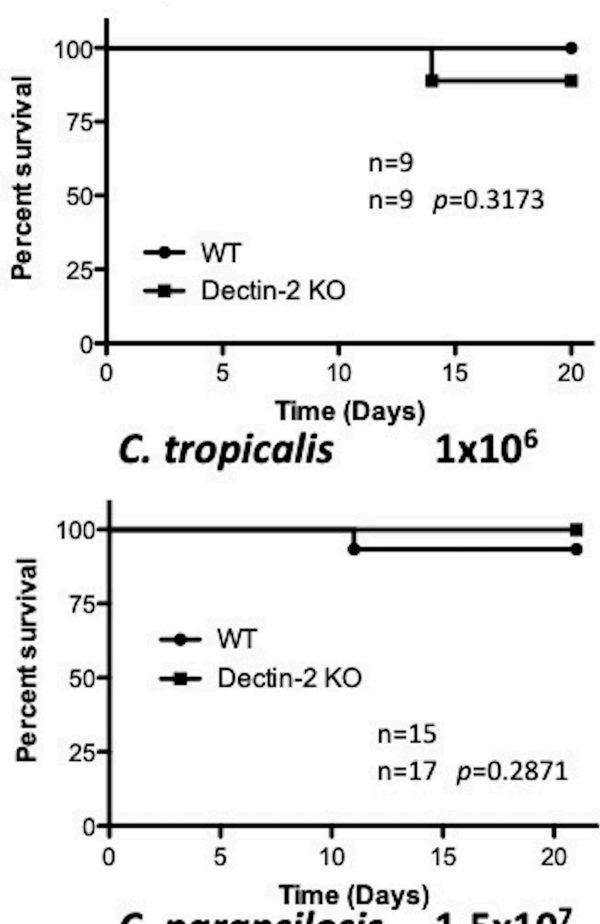

C. parapsilosis

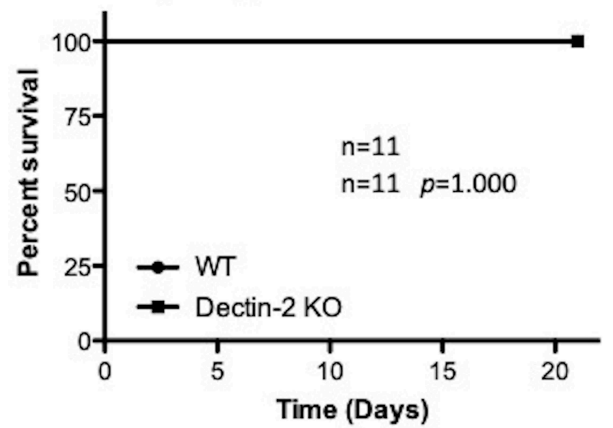

B

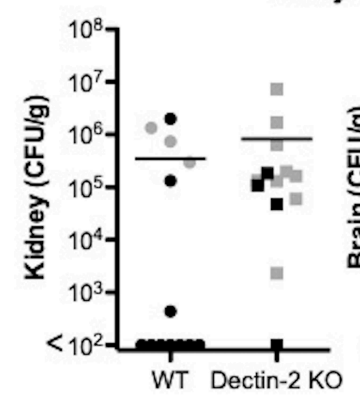

Day 30: $1.5 \times 10^{5}$

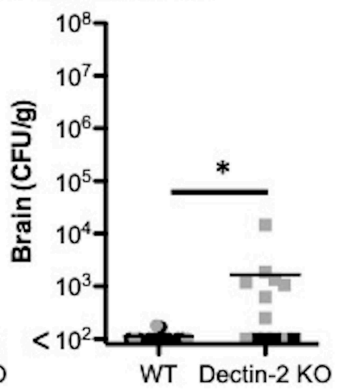

Day 20: $1.5 \times 10^{7}$
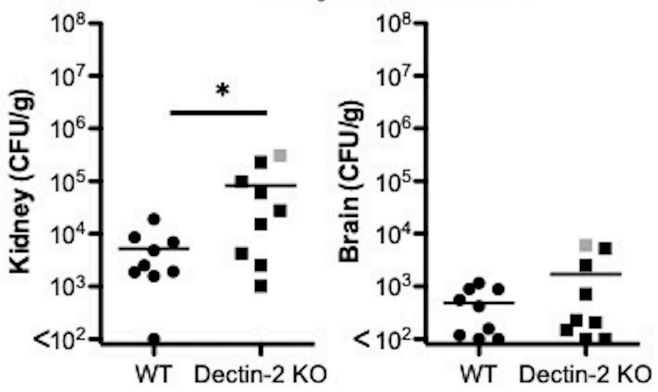

Day 21: $1 \times 10^{6}$
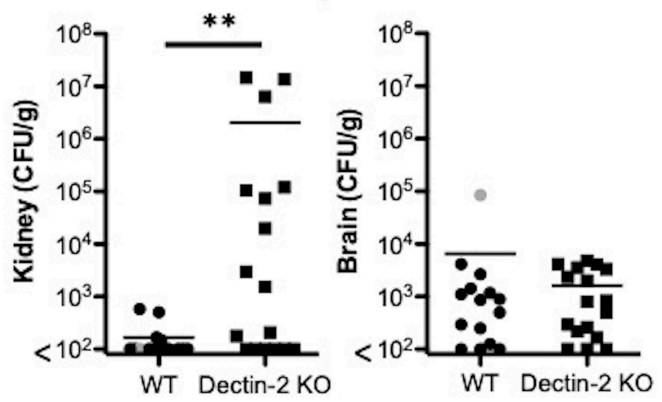

Day 21: $1.5 \times 10^{7}$
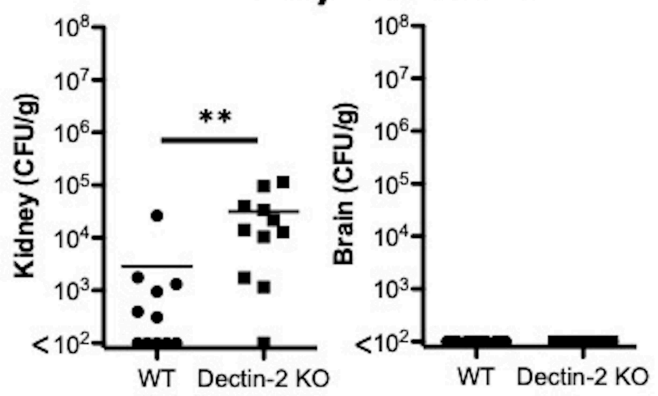

FIGURE 6 | Dectin-2 is important for clearance of multiple Candida spp. (A) WT and Dectin-2 KO mice were infected intravenously with the indicated doses of C. albicans for 30 days, C. glabrata for 20 days, C. tropicalis for 21 days, or C. parapsilosis for 21 days. Survival curves based on humane end-point of infected WT (filled circles) and Dectin-2 KO mice (filled squares). Graphs are the cumulative result of 2-3 independent experiments. Log-rank test. (B) CFU in the kidneys of WT and Mincle KO mice at 20-30 days after infection (black symbols) or at time of death by humane end point (gray symbols). Graphs are the cumulative result of 2-3 independent experiments. Each symbol represents an individual mouse. Mann-Whitney test on transformed data (C. albicans kidney and brain; C. tropicalis kidney and brain; C. parapsilosis kidney), Student's $t$-test on transformed data (C. glabrata kidney and brain), One sample t-test (C. parapsilosis brain). Nine out of nine WT mice in the $C$. glabrata graphs are the same as Figure $\mathbf{3}$ and 10 out of 14 WT mice in the C. tropicalis graphs are the same as Figure $\mathbf{4}$ of a previous publication from our group (Thompson et al., 2019). ${ }^{*} p<0.05,{ }^{* *} p<0.01$. 
clearance of four clinically relevant Candida spp. could be due to different mannan levels/structures on the cell wall. While we found significant differences in the mannan fibril length and phosphomannan in the four Candida spp., our results do not suggest a consistent relationship between type/amount of mannan and Mincle or Dectin-2 involvement in cytokine production or fungal clearance. Spatial orientation and exposure of different mannan ligands on the Candida cell wall was recently shown to greatly influence recognition by mannanbinding CLRs, including Dectin-2 (Vendele et al., 2020). It is possible that differences in cell wall architecture of the distinct Candida spp. tested here, could translate to different exposure of these ligands and greatly influence the ability of immune cells to recognize them via Mincle and Dectin-2. An in-depth analysis of the cell wall and the mannan structure on these Candida spp. may help to identify a pattern that could connect a specific mannan structure/type that is associated with each CLR, however, from our data, this pattern is not currently evident. Overall in this study, we have shown that Dectin-2 in particular, but also Mincle are important for Candida-mediated immune responses during systemic infection, although the level of CLR importance varies with species and likely strain of Candida.

\section{DATA AVAILABILITY STATEMENT}

The raw data supporting the conclusions of this article will be made available by the authors, without undue reservation.

\section{ETHICS STATEMENT}

The animal study was reviewed and approved by the Cardiff University's Animal Welfare and Ethical Review Body.

\section{AUTHOR CONTRIBUTIONS}

AT designed and performed the experiments. DF performed the experiments and wrote the manuscript. LW and JG performed the experiments. PT and NG guided the research. SO conceptualized and guided the research, designed and performed the experiments, and wrote the manuscript. All authors contributed to the manuscript revision, and read and approved the submitted version.

\section{REFERENCES}

Arendrup, M., Horn, T., and Frimodt-Møller, N. (2002). In vivo pathogenicity of eight medically relevant candida species in an animal model. Infection 30, 286-291. doi: 10.1007/s15010-002-2131-0

Ballou, E. R., Avelar, G. M., Childers, D. S., Mackie, J., Bain, J. M., Wagener, J., et al. (2016). Lactate signalling regulates fungal $\beta$-glucan masking and immune evasion. Nat. Microbiol. 2:16238.

\section{FUNDING}

SO was supported by a Sir Henry Dale Fellowship jointly funded by the Wellcome Trust and the Royal Society (Grant No. 099953/Z/12/Z) and by a Wellcome Trust Cross-Disciplinary Award. NG acknowledges Wellcome Trust support of a Senior Investigator (101873/Z/13/Z), Collaborative (200208/A/15/Z) and Strategic Awards (097377/Z11/Z) and the MRC Centre for Medical Mycology (MR/N006364/2). PT was funded by a Wellcome Trust Investigator Award (107964/Z/15/Z) and the UK Dementia Research Institute.

\section{ACKNOWLEDGMENTS}

We wish to acknowledge the NIH-sponsored Mutant Mouse Regional Resource Center (MMRRC) National System as the source of genetically altered mice (C57BL/6-Clec4etm1.1Cfg/Mmucd 031936-UCD) for use in this study. The mice were produced and deposited to the MMRRC by the Consortium for Functional Glycomics supported by the National Institute of General Medical Sciences (GM62116). We also thank the Microscopy and Histology Core Facility at the University of Aberdeen for expert assistance with TEM.

\section{SUPPLEMENTARY MATERIAL}

The Supplementary Material for this article can be found online at: https://www.frontiersin.org/articles/10.3389/fmicb. 2021.633229/full\#supplementary-material

Supplementary Figure 1 | Candida-induced responses in BMDMs are largely independent of Mincle and Dectin-2. (A-C) BMDMs from WT and Mincle KO mice $\mathbf{( A , C )}$ or from WT and Dectin-2 KO mice $\mathbf{( B , C )}$ were stimulated with $C$. albicans SC5314 (Ca), C. glabrata SCS74761 (Cg), C. tropicalis SCS74663 (Ct), and C. parapsilosis SCSB5882 (Cp) at a MOI of 1:1 (Candida sp.: BMDMs). Cytokine levels (A,B) and nitric oxide levels $\mathbf{( C )}$ in the supernatants were measured after 24 $\mathrm{h}$ incubation. Results are presented as means \pm SEM of 4-7 (A), 3 (B), or 3 (C) independent experiments (Two-way ANOVA with Bonferroni's post-test). I = Interaction between "Genotype" and "Candida" variables, G

(Genotype) = impact of BMDM genotype, C (Candida) = impact of different Candida spp. tested. (A) WT data is the same as $\mathrm{in}^{26}$, for 2 out of $4-7$ replicates for TNF, IL-12p40, IL-1 $\beta, I L-6$, and IL-10. ${ }^{\star} p<0.05,{ }^{\star \star} p<0.01,{ }^{* \star *} p<0.001$.

Supplementary Figure 2 | CLR Expression on BMDMs and BMDCs. Representative flow cytometry histograms showing basal Mincle and Dectin-2 surface expression and Mincle expression following $4 \mathrm{~h}$ LPS stimulation on BMDMs and BMDCs. Plots are representative of 2-3 independent experiments. Dashed line $=$ isotype control, solid line $=$ CLR.

Bongomin, F., Gago, S., Oladele, R., and Denning, D. (2017). Global and multinational prevalence of fungal diseases-estimate precision. J. Fungi 3:57. doi: 10.3390/jof3040057

Branzk, N., Lubojemska, A., Hardison, S. E., Wang, Q., Gutierrez, M. G., Brown, G. D., et al. (2014). Neutrophils sense microbe size and selectively release neutrophil extracellular traps in response to large pathogens. Nat. Immunol. 15, 1017-1025. doi: 10.1038/ni. 2987 
Brown, G. D., Denning, D. W., Gow, N. A., Levitz, S. M., Netea, M. G., White, T. C., et al. (2012). Hidden killers: human fungal infections. Sci. Transl. Med. 4:165rv13. doi: $10.1126 /$ scitranslmed.3004404

Brown, G. D., Taylor, P. R., Reid, D. M., Willment, J. A., Williams, D. L., Martinez-Pomares, L., et al. (2002). Dectin-1 is a major $\beta$-glucan receptor on macrophages. J. Exp. Med. 196, 407-412. doi: 10.1084/jem.20020470

Chen, S. M., Shen, H., Zhang, T., Huang, X., Liu, X. Q., Guo, S. Y., et al. (2017). Dectin-1 plays an important role in host defense against systemic Candida glabrata infection. Virulence 8, 1643-1656. doi: 10.1080/21505594. 2017.1346756

Denning, D. W., Kneale, M., Sobel, J. D., and Rautemaa-Richardson, R. (2018). Global burden of recurrent vulvovaginal candidiasis: a systematic review. Lancet Infect. Dis. 18, e339-e347.

Ferwerda, B., Ferwerda, G., Plantinga, T. S., Willment, J. A., van Spriel, A. B., Venselaar, H., et al. (2009). Human dectin-1 deficiency and mucocutaneous fungal infections. N. Engl. J. Med. 361, 1760-1767.

Gow, N. A. R., van de Veerdonk, F. L., Brown, A. J. P., and Netea, M. G. (2011). Candida albicans morphogenesis and host defence: discriminating invasion from colonization. Nat. Rev. Microbiol. 10, 112-122. doi: 10.1038/nrmicro2711

Guinea, J. (2014). Global trends in the distribution of Candida species causing candidemia. Clin. Microbiol. Infect. 20, 5-10. doi: 10.1111/1469-0691.12539

Haider, M., Dambuza, I. M., Asamaphan, P., Stappers, M., Reid, D., Yamasaki, S., et al. (2019). The pattern recognition receptors dectin-2, mincle, and FcR $\gamma$ impact the dynamics of phagocytosis of Candida, Saccharomyces, Malassezia, and Mucor species. PLoS One 14:e220867. doi: 10.1371/journal.pone.0220867

Hall, R. A., and Gow, N. A. R. (2013). Mannosylation in Candida albicans: role in cell wall function and immune recognition. Mol. Microbiol. 90, 1147-1161. doi: $10.1111 / \mathrm{mmi} .12426$

Hardison, S. E., and Brown, G. D. (2012). C-type lectin receptors orchestrate antifungal immunity. Nat. Immunol. 13, 817-822. doi: 10.1038/ni.2369

Hobson, R. P., Munro, C. A., Bates, S., MacCallum, D. M., Cutler, J. E., Heinsbroek, S. E., et al. (2004). Loss of cell wall mannosylphosphate in Candida albicans does not influence macrophage recognition. J. Biol. Chem. 279, 39628-39635. doi: 10.1074/jbc.m405003200

Ifrim, D. C., Bain, J. M., Reid, D. M., Oosting, M., Verschueren, I., Gow, N. A., et al. (2014). Role of dectin-2 for host defense against systemic infection with Candida glabrata. Infect. Immun. 82, 1064-1073. doi: 10.1128/iai.01189-13

Ifrim, D. C., Quintin, J., Courjol, F., Verschueren, I., van Krieken, J. H., Koentgen, F., et al. (2016). The role of dectin-2 for host defense against disseminated candidiasis. J. Interf. Cytokine Res. 36, 267-276. doi: 10.1089/jir.2015.0040

Kullberg, B. J., and Arendrup, M. C. (2015). Invasive candidiasis. N. Engl. J. Med. 373, 1445-1456.

Lamoth, F., Lockhart, S. R., Berkow, E. L., and Calandra, T. (2018). Changes in the epidemiological landscape of invasive candidiasis. J. Antimicrob. Chemother. 73, i4-i13.

Lee, R. T., Hsu, T. L., Huang, S. K., Hsieh, S. L., Wong, C. H., Lee, Y. C., et al. (2011). Survey of immune-related, mannose/fucose-binding C-type lectin receptors reveals widely divergent sugar-binding specificities. Glycobiology 21, 512-520. doi: 10.1093/glycob/cwq193

Lockhart, S. R. (2014). Current epidemiology of Candida Infection. Clin. Microbiol. Newsl. 36, 131-136. doi: 10.1016/j.clinmicnews.2014.08.001

Mccarty, T. P., and Pappas, P. G. (2016). Invasive candidiasis. Infect. Dis. Clin. NA 30, 103-124.

McGreal, E. P., Rosas, M., Brown, G. D., Zamze, S., Wong, S. Y., Gordon, S., et al. (2006). The carbohydrate-recognition domain of Dectin-2 is a C-type lectin with specificity for high mannose. Glycobiology 16, 422-430. doi: 10.1093/ glycob/cwj077

Netea, M. G., Gow, N. A., Munro, C. A., Bates, S., Collins, C., Ferwerda, G., et al. (2006). Immune sensing of Candida albicans requires cooperative recognition of mannans and glucans by lectin and Toll-like receptors. J. Clin. Invest. 116, 1642-1650. doi: 10.1172/jci27114

Nguyen, T. N. Y., Padungros, P., Wongsrisupphakul, P., Sa-Ard-Iam, N., Mahanonda, R., Matangkasombut, O., et al. (2018). Cell wall mannan of Candida krusei mediates dendritic cell apoptosis and orchestrates Th17 polarization pathway via TLR-2/MyD88-dependent pathway. Nat. Sci. Rep. $8: 17123$.

Orr, S. J., Burg, A. R., Chan, T., Quigley, L., Jones, G. W., Ford, J. W., et al. (2013). LAB/NTAL facilitates fungal/PAMP-induced IL-12 and IFN- $\gamma$ production by repressing $\beta$-catenin activation in dendritic cells. PLoS Pathog. 9:e1003357. doi: 10.1371/journal.ppat.1003357

Pappas, P. G., Lionakis, M. S., Arendrup, M. C., Ostrosky-zeichner, L., and Kullberg, B. J. (2018). Invasive candidiasis. Nat. Rev. Diease Prim. 4, 1-20.

Patin, E. C., Jones, A. V., Thompson, A., Clement, M., Liao, C. T., Griffiths, J. S., et al. (2016). IL-27 induced by select candida spp. via TLR7/NOD2 signaling and IFN- $\beta$ production inhibits fungal clearance. J. Immunol. 197, 208-221. doi: 10.4049/jimmunol.1501204

Patin, E. C., Thompson, A., and Orr, S. J. (2019). Pattern recognition receptors in fungal immunity. Semin. Cell Dev. Biol. 89, 24-33. doi: 10.1016/j.semcdb.2018. 03.003

Pfaller, M. A., and Diekema, D. J. (2007). Epidemiology of invasive candidiasis: a persistent public health problem. Clin. Microbiol. Rev. 20, 133-163. doi: 10.1128/cmr.00029-06

Plantinga, T. S., Hamza, O. J., Willment, J. A., Ferwerda, B., van de Geer, N. M., Verweij, P. E., et al. (2010). Genetic variation of innate immune genes in HIVinfected african patients with or without oropharyngeal candidiasis. J. Acquir. Immune Defic. Syndr. 55, 87-94. doi: 10.1097/qai.0b013e3181e53c64

Plantinga, T. S., van der Velden, W. J., Ferwerda, B., van Spriel, A. B., Adema, G., Feuth, T., et al. (2009). Early stop polymorphism in human DECTIN1 is associated with increased Candida colonization in hematopoietic stem cell transplant recipients. Clin. Infect. Dis. 49, 724-732. doi: 10.1086/ 604714

Pradhan, A., Avelar, G. M., Bain, J. M., Childers, D., Pelletier, C., Larcombe, D. E., et al. (2019). Non-canonical signalling mediates changes in fungal cell wall PAMPs that drive immune evasion. Nat. Commun. 10, 1-14.

Robinson, M. J., Osorio, F., Rosas, M., Freitas, R. P., Schweighoffer, E., Gross, O., et al. (2009). Dectin-2 is a Syk-coupled pattern recognition receptor crucial for Th17 responses to fungal infection. J. Exp. Med. 206, 2037-2051. doi: 10.1084/jem.20082818

Saijo, S., Ikeda, S., Yamabe, K., Kakuta, S., Ishigame, H., Akitsu, A., et al. (2010). Dectin-2 recognition of alpha-mannans and induction of Th17 cell differentiation is essential for host defense against Candida albicans. Immunity 32, 681-691. doi: 10.1016/j.immuni.2010.05.001

Sam, Q. H., Yew, W. S., Seneviratne, C. J., Chang, M. W., and Chai, L. Y. A. (2018). Immunomodulation as therapy for fungal infection: are we closer? Front. Microbiol. 9:1612. doi: 10.3389/fmicb.2018.01612

Sato, K., Yang, X. L., Yudate, T., Chung, J. S., Wu, J., Luby-Phelps, K., et al. (2006). Dectin-2 is a pattern recognition receptor for fungi that couples with the Fc receptor $\gamma$ chain to induce innate immune responses. J. Biol. Chem. 281, 38854-38866. doi: 10.1074/jbc.m606542200

Segal, B. H., Kwon-Chung, J., Walsh, T. J., Klein, B. S., Battiwalla, M., Almyroudis, N. G., et al. (2006). Immunotherapy for fungal infections. Clin. Infect. Dis. 42, 507-515.

Shibata, N., Suzuki, A., Kobayashi, H., and Okawa, Y. (2007). Chemical structure of the cell-wall mannan of Candida albicans serotype A and its difference in yeast and hyphal forms. Biochem. J. 404, 365-372. doi: 10.1042/bj20070081

Sun, J., Zhang, X., Broderick, M., and Fein, H. (2003). Measurement of nitric oxide production in biological systems by using griess reaction assay. Sensors 3, 276-284. doi: 10.3390/s30800276

Taylor, P. R., Gordon, S., and Martinez-pomares, L. (2005). The mannose receptor: linking homeostasis and immunity through sugar recognition. Trends Immunol. 26, 104-110. doi: 10.1016/j.it.2004.12.001

Taylor, P. R., Tsoni, S. V., Willment, J. A., Dennehy, K. M., Rosas, M., Findon, H., et al. (2007). Dectin-1 is required for $\beta$-glucan recognition and control of fungal infection. Nat. Immunol. 8, 31-38. doi: 10.1038/ni1408

Thompson, A., Davies, L. C., Liao, C. T., da Fonseca, D. M., Griffiths, J. S., Andrews, R., et al. (2019). The protective effect of inflammatory monocytes during systemic C. albicans infection is dependent on collaboration between C-type lectin-like receptors. PLoS Pathog. 15:e1007850. doi: 10.1371/journal. ppat. 1007850

Thompson, A., Griffiths, J. S., Walker, L., da Fonseca, D. M., Lee, K. K., Taylor, P. R., et al. (2019). Dependence on dectin-1 varies with multiple candida species. Front. Microbiol. 10:1800. doi: 10.3389/fmicb.2019.01800

van de Veerdonk, F. L., Netea, M. G., Joosten, L. A., van der Meer, J. W., and Kullberg, B. J. (2010). Novel strategies for the prevention and treatment of Candida infections: the potential of immunotherapy. FEMS Microbiol. Rev. 34, 1063-1075. 
Vendele, I., Willment, J. A., Silva, L. M., Palma, A. S., Chai, W., Liu, Y., et al. (2020). Mannan detecting C-type lectin receptor probes recognise immune epitopes with diverse chemical, spatial and phylogenetic heterogeneity in fungal cell walls. PLoS Pathog. 16:1007927. doi: 10.1371/journal.ppat.1007927

Walker, L., Sood, P., Lenardon, M. D., Milne, G., Olson, J., Jensen, G., et al. (2018), The viscoelastic properties of the fungal cell wall allow traffic of ambisome as intact liposome vesicles. mBio 9, 1-15.

Wells, C. A., Salvage-Jones, J. A., Li, X., Hitchens, K., Butcher, S., Murray, R. Z., et al. (2008). The macrophage-inducible C-type lectin, mincle, is an essential component of the innate immune response to Candida albicans. J. Immunol. 180, 7404-7413. doi: 10.4049/jimmunol.180.11.7404

Yadav, B., Mora-Montes, H. M., Wagener, J., Cunningham, I., West, L., Haynes, $\mathrm{K}$., et al. (2020). Differences in fungal immune recognition by monocytes and macrophages: N-mannan can be a shield or activator of immune recognition. Cell Surf. 6:100042. doi: 10.1016/j.tcsw.2020.100042

Yamasaki, S., Ishikawa, E., Sakuma, M., Hara, H., Ogata, K., Saito, T., et al. (2008). Mincle is an ITAM-coupled activating receptor that senses damaged cells. Nat. Immunol. 9, 1179-1188. doi: 10.1038/ni.1651

Yamasaki, S., Matsumoto, M., Takeuchi, O., Matsuzawa, T., Ishikawa, E., Sakuma, M., et al. (2008). C-type lectin mincle is an activating receptor for pathogenic fungus, Malassezia. Proc. Natl. Acad. Sci. U S A. 106, 1897-1902. doi: 10.1073/ pnas. 0805177106
Yapar, N. (2014). Epidemiology and risk factors for invasive candidiasis. Ther. Clin. Risk Manag. 10, 95-105. doi: 10.2147/tcrm. s40160

Zaoutis, T. E., Argon, J., Chu, J., Berlin, J. A., Walsh, T. J., Feudtner, C., et al. (2005). The epidemiology and attributable outcomes of candidemia in adults and children hospitalized in the united states: a propensity analysis. Clin. Infect. Dis. 41, 1232-1239. doi: 10.1086/496922

Conflict of Interest: The handling editor declared a shared affiliation with one of the authors JG at time of review.

The authors declare that the research was conducted in the absence of any commercial or financial relationships that could be construed as a potential conflict of interest.

Copyright (c) 2021 Thompson, da Fonseca, Walker, Griffiths, Taylor, Gow and Orr. This is an open-access article distributed under the terms of the Creative Commons Attribution License (CC BY). The use, distribution or reproduction in other forums is permitted, provided the original author(s) and the copyright owner(s) are credited and that the original publication in this journal is cited, in accordance with accepted academic practice. No use, distribution or reproduction is permitted which does not comply with these terms. 